Catherine CESSAC, Elisabeth Jacquet De La Guerre, Une femme compositeur sous le règne de Louis XIV, Arles, Actes Sud, 1995, 213 p.

\title{
Claire Bernard
}

\section{OpenEdition}

Journals

Édition électronique

URL : https://journals.openedition.org/clio/5032

DOI : 10.4000/clio.5032

ISSN : $1777-5299$

Éditeur

Belin

Édition imprimée

Date de publication : 1 avril 2007

Pagination : 249-290

ISBN : 978-2-85816-900-9

ISSN : 1252-7017

Référence électronique

Claire Bernard, "Catherine CESSAC, Elisabeth Jacquet De La Guerre, Une femme compositeur sous le règne de Louis XIV, Arles, Actes Sud, 1995, 213 p. », Clio. Histoire, femmes et sociétés [En ligne], 25 | 2007, mis en ligne le 03 octobre 2007, consulté le 24 avril 2022. URL : http://journals.openedition.org/ clio/5032 ; DOI : https://doi.org/10.4000/clio.5032

Ce document a été généré automatiquement le 24 avril 2022

Tous droits réservés 


\title{
Catherine CESSAC, Elisabeth Jacquet De La Guerre, Une femme compositeur sous le règne de Louis XIV, Arles, Actes Sud, 1995, $213 \mathrm{p}$.
}

\author{
Claire Bernard
}

1 Ces dernières années, plusieurs travaux sur l'histoire des femmes s'intéressent à leur présence et à leurs rôles dans les domaines artistiques laissés de côté par les ouvrages généraux plus anciens. Pour autant, des thèmes sont encore à explorer en ce qui concerne le rôle de certaines dans ces secteurs pour la période moderne. C'est le cas de la musique, pour laquelle des éléments sur la présence féminine sont décelables à travers des ouvrages sur l'art musical de cette période. Ainsi, l'ouvrage consacré à Elisabeth Jacquet de La Guerre, de Catherine Cessac, musicologue de formation, montre un intérêt tout particulier par la mise en lumière de cette figure féminine d'exception, et par les riches éléments historiques qui déterminent un contexte favorable particulier. Au travers de dix-sept chapitres, alternant biographie détaillée et explications des oeuvres de cette musicienne, cette étude pose la question de la légitimité de la participation féminine dans l'art musical de la seconde moitié du XVIIe siècle.

2 Dès le prologue, le point délicat du rapport des femmes à la musique et plus spécifiquement à la création artistique est souligné. À toutes les périodes, des femmes se sont exercées à la composition sans pour autant être reconnues. Le droit à l'interprétation vocale et instrumentale leur était permis parallèlement à l'éducation musicale qu'elles recevaient dans leur instruction. Toutefois, c'est en tant qu'amateur qu'elles sont menées à s'y intéresser, ce fait établissant des barrières à l'apprentissage de la théorie et de la création, comme le précise Pierre Maréchaux ${ }^{1}$. Dans ce contexte, les figures musicales féminines doivent souvent leurs succès à leur entourage familial, Elisabeth Jacquet de La Guerre en est un des exemples les plus marquants. 
3 Les premiers chapitres permettent une mise en contexte de ce parcours atypique. Elisabeth fut éduquée par son père et bénéficia d'un apprentissage au foyer. Cet aspect est primordial et se retrouve dans de nombreux milieux artistiques. Dans des cas comme celui-ci, plus qu'une éducation «domestique », c'est d'une transmission du savoir familial dont hérite la jeune fille. L'apprentissage pouvait s'étendre à la descendance féminine, et la volonté paternelle donnait également accès à des réseaux de relations qui l'introduisaient dans ce milieu relativement clos. Ce facteur est d'autant plus déterminant que l'endogamie était chose courante au sein des milieux professionnels, et donnait une impulsion ainsi que des soutiens à l'insertion. Elisabeth Jacquet se marie avec Marin de La Guerre, issu du même cercle professionnel et choisit de garder son nom de jeune fille et d'épouse; elle cumulait, par cette «double identité » (p.21), la renommée de ces deux familles de musiciens et les réseaux qui s'y rattachaient. Cette intégration lui donnait l'opportunité de démontrer son talent et de créer des liens professionnels avec les plus grands tels les Couperin. Dès son plus jeune âge, présentée à la cour par son père, son talent est reconnu en tant qu'interprète. Plus encore quand elle passe à ses premières compositions puis à la publication en 1687 de son Premier livre de Pièces de clavecin. Elle allie alors savoir-faire et théorie, connaissance et talent qu'elle démontre par la suite par sa volonté de s'exercer dans tous les registres : ballet, sonates, cantates religieuses puis profanes, jusqu'à un opéra comique.

D'un point de vue musicologique, auquel Catherine Cessac consacre de nombreuses pages, l'analyse de ses oeuvres prouve son rôle actif dans la musique du XVIIe siècle ainsi que l'aspect novateur de ses créations. Elle acquit donc une légitimité à part entière dans cette profession dans le cadre d'une carrière indépendante, acquisition que l'auteur lie aussi à sa personnalité particulière et à sa liberté d'esprit, renforcée par les vingt cinq ans d'autonomie qu'elle connut avec son veuvage. Son exemple peut donc s'inscrire dans les aspects généraux de l'apprentissage informel des filles et d'une insertion facilitée par des réseaux; il ne faut pas, en revanche, omettre que son talent indéniable et sa volonté de s'affirmer en tant que compositrice sont par la suite la source de la reconnaissance qu'elle acquit; Catherine Cessac nous fait part de la considération des contemporains grâce à de nombreuses citations à l'instar des éloges du Mercure Galant, de Titon du Tillet dans son Parnasse françois, ou les commentaires des autres compositeurs.

5 L'ouvrage met aussi le doigt sur certaines limites de la création féminine : son opéra, Céphale et Procris fut la première tragédie lyrique composée par une femme pour l'Académie Royale; cependant Catherine Cessac nous invite à nous interroger sur le lien possible entre son insuccès et son statut de femme. Dans cette optique, nous pouvons penser qu'il existerait une forme de résistance de la part du public, malgré l'acceptation de la création féminine que l'on retrouve aussi chez les peintres et sculpteurs. Ce phénomène détermine l'évolution contrastée, entre avancées et restrictions, de la place des femmes dans la culture du XVIIe siècle. Elisabeth Jacquet ne parvient pas non plus à un titre ni à une charge en tant que compositrice (p. 60) malgré l'importance de son oeuvre, alors que plusieurs sont accordées à des femmes s'exerçant dans l'interprétation de l'art musical sous Louis XIV. La liberté dont elle a pu jouir, tant d'un point de vue personnel qu'artistique, était donc ancrée dans les carcans maintenus à destination de "l'autre sexe ». Cependant, cette étude met en avant un bel exemple des possibles accessions des femmes à des carrières musicales et créatrices au cours du 
XVIIe siècle, ainsi que les conditions dans lesquelles l'insertion féminine était réalisable et remarquable, en attendant une synthèse consacrée à leurs activités et à leur place dans ce domaine.

\section{NOTES}

1. P. Maréchaux, « Savoir des doigts, savoir des voix : Les femmes et la pédagogie du clavecin (1630 à 1740)", in C. Nativel (e.a.), Femmes savantes, savoir des femmes, Du crépuscule de la Renaissance à l'aube des Lumières, Actes du colloque de Chantilly de 1995, Genève, Droz, 1999, p. 69-85. 